\title{
Cross-Cultural Research Methodology as an Inquiry in the Practices of Traditional Health Practitioners for Social Behavioural Change
}

\author{
Ramodungoane Tabane \\ Dept of Psychology of education \\ College of Education, University of South Africa \\ Tabanri@unisa.ac.za
}

Doi:10.5901/mjss.2013.v4n3p729

\begin{abstract}
Traditional health practicing, as a practice of tradition and culture, is a sensitive area for researchers and needs attention. Conducting research in this field brings with it many challenges as traditional health practitioners in most cases prefer to guard their trade and do not easily allow sojourners to enter this space. In this study, a qualitative research methodology was employed to gain critical insight on how traditional health practitioners and their patients articulate, understand and act relative to the concepts of disease risk and prevention to their patients. To gather the emic insight of the traditional health practitioners, focus groups and in-depth individual interviews were facilitated by the traditional health practitioners as moderators and interviewers. The study indicated that with desirable research methodology, positive and insightful information can be elicited from the closely guarded emic or idiographic practices.
\end{abstract}

Keywords: Cross-cultural; Research Methodology; Traditional Health Practitioner; Culture; Emic; Etic; Psychology

\section{Introduction}

Research methodology is used to collect data for various reasons. Data collection is a sensitive area of research as it is almost impossible to collect data in a homogenous or isotropic situation. This is so because people have different traits, experiences and cultures and thus there are more cross-cultural researches conducted than envisaged. However, for the collection of authentic data in a cross-cultural setting, the practitioner needs to be aware of the research setting, the participants and the data that is being collected especially in sensitive issues. Sekaran (1983) noted that cross-cultural research is still in its infancy and should be allowed to explore and discover rather than be stifled by superimposed constricting research design and rigour. Two decades post the statement, it is still true.

Due to contestation of the term its self, study of culture requires deeper understanding of the term and its fluidity as it might be linked to aspects such as race, ethnicity and language and sub-cultures within a particular community. ÆÉgisdóttir, Gerstein and Çinarbaş (2008) argue that when studying cultural issues we need to look at three approaches as noted by Triandis (2000), indigenous, the cultural and the cross-cultural approaches. The indigenous and cultural speaks to the emics or "uniqueness" to a culture while cross-culture focuses on the etics or factors common acrosscultures. Égisdóttir et al. (2008: 190) state that, in cross-cultural approach, "the goal is to understand similarities and differences across cultures and the comparability of cross-cultural categories". Thus, conducting research in a crosscultural setting is an art and science that requires researchers to be in-tune and aware of their status as amongst others as sojourners and not the member of the concerned community, in terms of cultural practices for instance, even though they might be of the same race and/ or culture.

This paper addresses the research methodology in an exploratory study of how Traditional Health Practices (THP) in South Africa communicates the concepts of disease risk and prevention to their patients. It looks at the research methodology employed with the custodians of cultural practices, the THPs to gain critical insight of the phenomena under investigation. THPs play an important role as the primary of the primary health care in South Africa and the sub-Saharan Africa. THPs are repositories of extensive and untapped traditional knowledge about sensitive issues (Broad Reach Health Care, Tabane, Mbele-Khama and Dlamini, 2012). This practice cannot be wished away and despite availability of western scientific practices, the THP practices still receive a volume of clients in search for treatment before they themselves are in some cases referred to allopathic medicine by the THPs. There is a need to understand this tradition, culture and practice. According to Deputy Minister of Health, Dr. Gwen Ramokgopa, the role of THPs in the promotion of health could not be denied and their influence in society could not be ignored (e-Bulletin, 2013). However, the THPs guard their practice and anecdotal evidence indicates that due to lack of trust, they do not allow outsiders, in this 
instance, non-THPs access into this age long traditions of helping people. Researchers are seen as exploiters, they want to learn the THP practice, especially medicine and patent and commercialise it without them, as people with indigenous knowledge earning and gaining from such exercises. Webster and John (2010: 175) alert that:

\begin{abstract}
"researchers [are] faced with confronting the history of exploitive research that contributed little to no benefit, or worse, research that caused damaging effects for Indigenous peoples and their communities can meet with resistance and even be barred from communities whose histories include such experiences. Even those Indigenous communities that allow entry into the field often approach these research 'collaborations' with distrust or apprehension".
\end{abstract}

Thus, there is a need for better and somewhat innovative methodologies that will allow researchers learn and make this age old tradition with wealth of information know and understood in the public domain as they might shed a light in different aspects of health, including mental health. The focus of this paper is not on the results of the study, but the methodology used and whether authentic data were gathered, analysed and reported. It discusses the methodological benefits and challenges when the researchers during cross-cultural researches choose to put themselves in the background and allow the emic or according to Sekaran (1983) 'idiographic' custodians to collect empirical research data. Hence, as noted from ÆÉgisdóttir et al. (2008), this paper is about etics or the cross-culture of the phenomenon of study. It is about whether the emic collection of data by the indigenous and/ or cultural custodian, THPs will yield authentic rich data.

\title{
Methodological Challenges and Consideration in Cross-Cultural Research
}

In their study, Webster and John (2010) focused on two important themes concerning cross-cultural research which are rooted in ethical dilemmas regarding cross-cultural research. As they observe, "these dilemmas involve interrelated dualistic relationships embedded in the western academic tradition, namely (a) the notion of insider and outsider in terms of ethnographic research in general and critical ethnographic research in particular; and (b) the notion of 'the academy' and the power relations between coloniser/ theoriser/ knowledge producer and Indigene/theorised/knowledge receiver" (Webster and John, 2010:187). The dualistic role involves the power relations amongst others that can be at play in the cross-cultural research. It involves for instance the western knowledge (well researched) being superimposed on the indigenous (less researched) fields and thus silencing the traditions of certain communities. Dualistic role in terms of the insider/ outsider relationship is an important element in cross-cultural research because, in terms of the emics or the etics as used in these researches, information can be lost or even distorted if the roles are not clearly outlined.

As noted by Sekaran (1983: 61), there are five major methodological and design concerns in cross cultural research, namely, ensuring functional equivalence, problems of instrumentation, data-collection methods, sampling design issues and data analysis. KÉgisdóttir et al. (2008) add bias and translations to the list as other aspects to be considered in cross-cultural research. Challenges in cross-cultural research as highlighted by Æ̇́gisdóttir et al. (2008: 191) refer to "any cross-cultural comparison of psychological constructs (within [e.g., ethnic groups] and between countries) and emphasises that these challenges are greater, though, in cross-cultural comparisons requiring translation of instruments."

According to ÆÉgisdóttir et al. (2008), equivalence is a key concept in cross-cultural psychology. To ensure functional equivalence, notes Sekaran (1983: 62), valid cross-cultural behaviour could be made when the phenomenon in study has been developed in different cultures in response to similar problems shared by different social or cultural groups. There are several explanations of equivalence defined by different experts. Sekaran (1983: 62), on the other hand, exerted that

\footnotetext{
"in developing proper instrument to be used in cross-cultural research, several types of equivalence should be considered and they include, vocabulary equivalence or a translation that is equivalent to the original language in which the instrument was developed; idiomatic equivalence which could become a serious problem when some idiom unique to one language just cannot be translated properly in other languages; grammatical and syntactical equivalence, which is especially important when translating long passages; experiential equivalence or the equivalence of the inferences drawn by the respondents in various cultures and finally, conceptual equivalence, where the meaning of certain concepts such as love may differ in different cultures."
}

Lonner (1985) mentions four types of equivalence, namely, functional, conceptual, metric and linguistic; while Van de Vijver and Leung (1997) explains equivalence in terms of hierarchical order between lower and higher degree of equivalence and these are also divided in four categories, that is construct non-equivalence, construct equivalence, measurement-unit equivalence and scalar equivalence. With regard to data collection, Sekaran (1983: 63) mentions that 
there are four important issues, namely, response equivalence, timing of data collection in different cultures, status and other psychological issues, especially in developing countries; and cross-sectional versus longitudinal data collection. The response equivalence can be ensured by adopting uniform data collection procedures in all the cultures in which a problem is being investigated.

The timing of data collection from the different countries is also important. Too much time should not elapse between the periods when data are collected from different countries. In terms of the Status and other critical psychological issues in data collection, Mitchell (1969 in Sekaran 1983: 64) mentions that

\begin{abstract}
in interview surveys, the equalitarian-oriented interviewing techniques used in the West may not be appropriate in societies which have sharp status and authority cleavages. In the same vein, when a foreigner comes and collects data, or when a local citizen who is a permanent resident of another country collects the data to be analysed and published abroad, respondents may fear that their country will be portrayed in an inferior light.
\end{abstract}

These issues pose additional concerns for cross-cultural researchers.

Bias is an important aspect of consideration in research in general. According to ḰEgisdóttir et al. (2008), sources of bias are construct, method and item bias. Construct bias occurs when the whole measured is not identical across cultural group; method bias may come from the characteristics of the instrument or from its administration while item bias refers to measurement at the item level which might have resulted for instance from poor translation or poor item formulation. Possible sources for method bias amongst many might be "response styles; communication problems between investigators and participants, and differences in physical conditions under which the instrument is administered across cultures" (KÉgisdóttir et.al (2008: 194). According to Sekaran (1983), sampling concerns with regard to crosscultural sampling design includes basic issues as whether we can possibly obtain samples that are representative of the culture and whether the sample constitutes a valid sample.

\title{
Methodology
}

The ethical clearance was applied for and granted before this study could take place. A qualitative research methodology was employed in this study, that is, an exploratory study of how Traditional Health Practices (THP) in South Africa communicates the concepts of disease risk and prevention to their patients. The study was concerned with the communication of concepts of disease between western and traditional practices. Thus, as noted by Ǽgisdóttir et al. (2008) once a cross-culture phenomenon has being identified, it is equally important to establish how the construct will be studied and the type of bias expected.

Method bias was one of the methodological consideration in this study, thus the usage of the THPs as moderators; interviewers and facilitators for gathering data.

According to ǼEgisdóttir et al. (2008: 196), in order to address method bias in cross-cultural research, the "researcher may need to rely on emic approaches (indigenous or cultural), develop measures meaningful to the culture and use culture-sensitive methodologies" an approach or technique noted by ǼEgisdóttir, et.al. (2008) as the assembly approach from Van de Vijver and Leung (1997). The dualistic power in terms of the being in the inside as well as outside of the phenomenon under study, was thought to be putting the study in the advantaged position because the participants might relate better with some of their own and at the same time THPs as research team members will give amongst other proper guidance to the entire team; language and concepts used will be captured by their first practitioners.

\subsection{Recruitment of facilitators, focus group moderators and in-depth interviewers}

The research team consisted of a technical team comprising of non-THP who was an educational psychologist and principal investigator; two non-THPs who were Broad Reach Health Care (BRHC) employees and were project leaders; two senior THPs who were co-investigators of the study. It was a strategic and conscious plan to involve the THPs because the principal investigator and the project leaders undertaking the study alone would have raised ethical and methodological issues in cross-cultural research. The THPs also doubled up as expert advisors on the study and facilitators during the research so all the THP language issues and practices can be respected and the non-THP do not behave in the manner that might upset or pollute the THP processes with their sojourner practices and thus offend the participants and three Traditional Health Practitioners who were the moderators and interviewers.

The involvement of the indigenous people in this study was complemented by the THPs and was further designed to address ethical concerns about power relationships that might serve to subordinate indigenous epistemology, methodology and representations of knowledge to the established western academic traditions (Webster and John, 
2010). Therefore, there were five THP practitioners in the study and they were of both genders (two males and three females). The experience range covered from 3 to more than 10 years. The two senior THPs (male and female) have been practicing for more 10 years and they have trained a number of new THPs and they participate in various THP forums and also involved in the legislation of the THPs in South Africa.

\subsection{Site}

Selecting research site is important and in a cross-cultural research, this becomes paramount as an element of an etic phenomenon must be present and distinct. The socio-economic status where THPs operate was considered thus rural and urban setting had to be represented. There were two provinces selected for this study. One was predominantly urban while the other was predominantly rural. Thus, there were four sites for the study. A pilot study was also conducted in a different province and it too hosted two sites. The research sites had to have THPs and the traditional practices must be active, that is, the communities must be using their services.

\subsection{Sample}

In South Africa, there are various THP committees within municipalities that are organised as forums and each forum holds a database of practicing THPs within their sub-districts. A letter of invitation explaining the study and the purpose and the purposive sampling method of the study was sent to the chairperson and secretary of each THP forum of the selected sites in the identified provinces. The forums were asked to identify 10 THPs from their database that met the selection criteria outlined in the letter. The forums were also asked to identify additional five as reservist in case challenges are experienced with any of the 10 identified to participate in the study.

There were two types of participants that were purposively selected to participate in the focus groups, namely, the THPs and the patients being treated by the THPs. For the selection of the THPs firstly, the Traditional Health Practitioners Act No. 22 of 2007 was used to select the types of THPs to participate in the study. The Act recognises four categories of THPs, namely diviners, herbalists, traditional birth attendants and traditional surgeons. Secondly, the THP experience was taken into consideration, thus the sample had to comprise the THP who has recently finished their apprenticeship (within the last two years) and those that have been practicing for more than two years. Thirdly, the sample had to have THP trainers or tutors. Fourthly, two issues were further important for representivity, that is age (above 18 years) and gender.

The second type of participants, that is, the patients treated by the THPs only had to meet the fourth purposive sampling dimension of the THPs, that is both genders and 18 years or older has to be present. However, the patients' purposive sampling dimensions were also that they had to be treated by the THPs from the four categories and at least five of them must be treated or counseled by a THP for HIV (the status was disclosed only to the interviewer and not the group). Thus, 80 participants, that is, 40 THPs and 40 patients were recruited through the THPs local and national associations within each sub-district to participate in the focus groups. That is 10 participants per site.

The study also conducted individual interviews with five participants from each of the four sites. Thus, 20 in-depth interviews were conducted and the purposive sampling dimension for individual interviews with THPs was that at least three categories of the THPs are present (however the two that must be there were diviners and herbalist); there should a traditional tutor and both genders must be represented. For the individual interviews with the patients, the sample had to at least comprise patients treated by at least three categories of the THPs (however the two that must be there were diviners and herbalist); both genders must be represented and at least two patients that are HIV positive should be selected.

\subsection{Data collection}

The focus group and interviews were conducted, recorded electronically and transcribed by the THPs. This was done so that information, subtle messages and the rich contexts of the responses are not lost in translation when done by nonTHPs. This was undertaken as measures to ensure trustworthiness and authenticity of the data collected and to be analysed.

\subsection{Data analysis}

The two project managers from BRHC were trained in the usage of computer software called Nvivo9, which is qualitative 
data analysis software. Nvivo9 was used to thematically analyse text from the study. The research team, firstly had to develop themes from the objective of the study and secondly, the THPs had to sensitise and advise the team on themes that are of THP nature and significant, thus developing such as themes for analysis. Transcripts were coded into broad categories and then supplemented by more coding sessions with the aim of allowing the data to guide coding rather than impose a complete coding scheme.

To ensure that the coding of the themes are somewhat in agreement by all members, the team members had to work individually on the same transcript, individually code it as per agreed themes and then inter-coder consensus had to be reached by team (both etic and emic) members. THP members of the research team and their knowledge in the field had to check and agree on the coded data. To ensure data quality, one THP member coded the original transcripts in his local language, thus ensuring that the translated and original transcripts receives equal rigour of coding and this also assisted in ensuring that message and richness of text was not lost in translation.

\subsection{Results}

After the study was conducted, various stakeholders meeting were hosted because the team wanted to use various platforms to evaluate the findings of the study before a final report was written. Therefore, peer group evaluations were conducted with researchers conducting similar types of studies with THPs in various contexts. Furthermore, a form of member checking was also conducted with the THPs who were not part of the study but participated somewhere in the process of the study through various stakeholders' meeting prior and post data collection. They were asked to review the preliminary report so that further consensus could be obtained and also to look for further avenues to moving forward.

\section{Discussion}

Conducting research in a cross-culture setting is a challenging task especially with the essence and elements that define a cross-cultural setting being fluid and thus requires a sensitive sharp understanding. The contestation of what is culture and the implied expectation of science on the concrete, challenges fields like cross-cultural psychology when embarking on cultural studies. Culture is explained differently and experienced diffidently. As noted, when studying cultural issues, according to Triandis (2000), we need to look at the indigenous, the cultural and the cross-cultural approaches. The Indigenous and cultural speaks to the emics or "uniqueness" to a culture while cross-culture focuses on the etics or factors common across-cultures.

The uniqueness of this study was in the understanding of the concepts of addressing and dealing with a particular phenomena and how to gather data from custodians of such knowledge, the THPs. However, it was important to note that a researcher and non-THPs involvement in the study brings the element of cross-culture that needs to be acknowledged that such involvement and/ or participation will have a bearing on the data collected. Furthermore, the dualistic position that the THPs played in this study, which is THPs as insiders (emics) and outsiders (etics), is a possible methodology that might need further investigation. In other words, researchers, especially those conducting cross-cultural research, need to be alert of their position as they actually might play the roles interchangeably within the methodological process which will have its own criticisms and issues to address. However, for a Black, Mopedi, and South African Male for instance, conducting a study on Black, isizulu culture about a women phenomenon and not realising that such a study in its design is cross-culture, it is erroneous.

According to Sekaran (1983) and Égisdóttir et al. (2008), there are methodological and design concerns that cross-cultural research should consider. The elements of equivalence should be taken consideration. To address vocabulary equivalence or to ensure translation that is equivalent to the original language, the THPs as the custodian of the phenomena under study were consulted. The THPS were fluent in the indigenous languages, conducted the data collections in required spoken languages. Furthermore, the THPs were conducting focus groups and individual interviews and so ensuring that issues that can arise as a result of idiomatic equivalence were addressed. The involvement of THPs as facilitators, moderators and interviewers also addressed the grammatical and syntactical equivalence, which is especially important when translating long passages as highlighted by Sekaran (1983).

It is also of paramount importance to note that the involvement of the custodian of traditional practices also sensitised the study to what Sekaran (1983) refers to as experiential equivalence or the equivalence of the inferences drawn by the respondents in various cultures and finally, conceptual equivalence, where the meaning of certain concepts such as love may differ in different cultures. The THPs, who were part of the study, collected and analysed data work with the concepts under of a particular culture in their daily dealings. Thus, their inside knowledge about the culture of the THP 
also contributed in ensuring that the data gathered and subsequently, analysed and reported is a true reflection and there is a minimal distortion of the respondents meanings and other traditional concepts.

Conducting cross-cultural study asks for researchers to be aware of their own bias. There was no construct bias as identified by KÉgisdóttir, et.al. (2008) because the THPs culture and phenomena being studied were explored by the people with the emic status of that particular study thus the whole that was being studied was identical across cultural group (THPs). The challenge was of construct bias as a result of the non-THPs. However, this was addressed by the involvement of the THPs as facilitators and their guidance of the entire study. One other bias that the cross-cultural researcher has to be vigilant of is method bias. To address method bias in cross-cultural research the "researcher may need to rely on emic approaches (indigenous or cultural), develop measures meaningful to the culture and use culturesensitive methodologies" (Ǽ́gisdóttir et al. 2008: 196).

In this study, the facilitators, moderators and interviewers had to take extra effort to ensure that they were not bias or their bias does not affect the data collected and analysed. The reason is bias can easily slip through in the data since the THPs were dealing with the phenomena and concepts foreign to the non-THPs especially in instances of translation and concept and question formulation. Thus, member checking during and after data collection was conducted. The THPs had to individually translate the transcripts and put them under discussion to ensure that the concepts, meaning and so forth were not distorted. The age ranges and practicing experience for both the respondents and the facilitators or interviewers also played a significant role in indicating that a broad spectrum of issues can be covered and thus there was match between for instance a young THP respondent in terms of his/ her pressing issues about the phenomena under study and the facilitator or the interviewer.

Sampling in this study was conducted in a manner that the researchers would not have an influence. An invitation letter for participants was sent to various THP organisations. The letter indicated the guidelines that had to be met so that there is representivity as desired by the study. Sekaran (1983) asserts that concerns with sampling in cross-cultural sampling design include, among other things, such basic issues as whether we can possibly obtain samples that are representative of the culture. The participants who participated in this study met the study sampling criteria, for instance, of having all the four categories of the THPs as recognised by the Traditional Health Practitioners Act No. 22 of 2007. Additional and on standby participants were also selected for the study in case any unfortunate circumstance rises or for instance as the ethical clearance guaranteed withdrawal of any active participants should they need to.

\section{Conclusions}

Some of the benefits of a well-designed cross-cultural research are that there will be more researchers with methodologies that are well researched and thus promote rigour in cross-cultural research. This also will filter in crosscultural disciplines such as psychology, counselling and assessment. According to ḰEgisdóttir et al. (2008: 216), "without strong cross-cultural methodology, erroneous conclusions can be made about similarities and differences between cultural groups in research and when using counselling and assessment strategies." Cross-cultural researchers should strive towards ensuring that their research methodology observes equivalence; bias; sampling that ensures cross-cultural representivity; and data collections are appropriate and gather the data that is trustworthy and analysis is free of emic and etic researcher bias. Researchers as experts in different fields should be comfortable in letting go of their power when conducing cross-cultural research. In this study, THPs were part of the research team. They were trained as facilitators, moderators and interviewers, thus skills were imparted to them and they also taught the researchers who were non-THPs about methodologies of conducting research in this closely guarded sector.

\section{References}

Broad Reach Health Care, Tabane, R., Mbele-Khama, S. and Dlamini, N. (2012). An exploratory study of how Traditional Health Practices (THP) in South Africa communicate the concepts of disease risk and prevention to their patients. REPORT.

Lonner, W. J. (1985). Issues in testing and assessment in cross-cultural counseling. The Counseling Psychologist, 13: 599-614.

Sekaran, U. (1983). Methodological and Theoretical Issues and Advancements in Cross-Cultural Research. Journal of International Business Studies. Special Issue on Cross-Cultural Management. 14 (2): 1-73.

The e-Bulletin of Health Profession Council of South Africa. (2013). Traditional healers and their medicine to be formalized. http://www.hpcsablogs.co.za/traditional-healers-and-their-medicine-to-be-formalised/ retrieved on 28 March 2013 at 16:15.

Triandis, H. C. (2000). Dialectics between cultural and cross-cultural psychology. Asian Journal of Social Psychology, 3: 185-195.

Van de Vijver, F. J. R., and Leung, K. (1997). Methods and data analysis for cross-cultural research. Thousand Oaks, CA: Sage.

Webster, J. P. and John, T.A. (2010). Preserving a space for cross-cultural collaborations: an account of insider/ outsider issues. Ethnography and Education, 5(2): 175-19.

Ǽ́gisdóttir, S, Gerstein, L. H. and Çinarbaş, D. C. (2008). Methodological Issues in cross-cultural counseling research: Equivalence, Bias and Translations. The counseling psychologist. Vol. 36 (2): 188-219. 